\title{
Purificação e Organofilização em Escala Piloto de Argilas Bentoníticas com Tensoativo Não lônico e Aplicação em Nanocompósitos Poliméricos
}

\author{
Wilma Sales Cavalcanti, Gustavo de Figueiredo Brito, Pankaj Agrawal, \\ Tomás Jeferson Alves de Mélo, Gelmires de Araújo Neves \\ Departamento de Engenharia de Materiais, Universidade Federal de Campina Grande - UFCG \\ Mísia Macedo Dantas \\ Departamento de Engenharia de Produção, Universidade Federal de Campina Grande - UFCG
}

\begin{abstract}
Resumo: $\mathrm{O}$ objetivo deste trabalho foi a purificação e organofilização em escala piloto de dois tipos de argila bentonita (cinza e verde) com tensoativo não iônico e a aplicação destas argilas em nanocompósitos com matriz de Polipropileno (PP). As argilas foram purificadas e em seguida organicamente modificadas com tensoativo não iônico tornandose organofílicas, sendo denominadas de CPO e VPO respectivamente, e caracterizadas por Análise Granulométrica por Difração a Laser (AG), análise química por Fluorescência de Raios X (EDX), Difração de Raios X (DRX) e espectroscopia no infravermelho (IV). Os resultados de AG e EDX mostraram redução significativa do teor de areia e da sílica livre na forma de quartzo confirmando que houve a purificação da argila. Os resultados de DRX e IV mostraram que a organofilização em escala piloto das argilas foi realizada com sucesso. Os compósitos contendo 3 pcr de argila foram caracterizados por DRX, Calorimetria exploratória diferencial (DSC), Termogravimetria (TG) e propriedades mecânicas. As análises por DRX indicaram formação de um nanocompósito com estrutura intercalada para o sistema PP/E-GMA/CPO, enquanto para o sistema PP/E-GMA/VPO formou-se um micro-compósito. Os resultados de DSC indicaram que as argilas não influenciaram na temperatura de fusão do PP. Os resultados de TG indicaram que as argilas CPO e VPO melhoraram a estabilidade térmica do PP. O sistema PP/E-GMA/CPO foi o que apresentou maior estabilidade térmica e maior módulo. As resistências à tração e ao impacto dos compósitos não apresentaram melhora significativa em relação ao PP puro. Mesmo assim, este comportamento é importante uma vez que as propriedades mecânicas do PP não foram deterioradas.
\end{abstract}

Palavras-chave: Nanocompósitos, organofilização, tensoativo não iônico, argila purificada.

\section{Purification and Organophilization in Pilot Scale of Bentonitic Clays with a Non-ionic Surfactant and their Application in Polymer Nanocomposites}

\begin{abstract}
The aim of this work was the purification and organophilization on a pilot scale of two types of bentonite clay (green and grey) with nonionic surfactant, and their application in nanocomposites with a Polypropylene (PP) matrix. The bentonitic clays were purified and then organically modified with a non-ionic surfactant to become organophilic, being referred to as CPO and VPO respectively, and characterized by Particle Size Analysis by Laser Diffraction (AG), chemical analysis by X-Ray Fluorescence (EDX), X-ray diffraction (XRD) and infrared spectroscopy (IR). The results of AG and EDX showed significant reduction in the amount of sand and free silica in the form of quartz confirming that the clay was purified. The results of XRD and IR showed that the organophilization on pilot scale of the clays was successful. The composites containing $3 \mathrm{phr}$ of clay were characterized by XRD, differential scanning calorimetry (DSC), thermogravimetry (TG) and mechanical properties. XRD results indicated that for the PP/E-GMA/CPO system a nanocomposite with an intercalated structure was formed. As for the PP/E-GMA/VPO system, a micro-composite was obtained. DSC results indicated that CPO and VPO clays did not affect the melting temperature of PP. TG results indicated that the CPO and VPO clays improved the thermal stability of PP. PP/E-GMA/CPO system showed the highest thermal stability. The mechanical properties of PP were not significantly improved with the addition of clay and compatibilizer. However they were not deteriorated.
\end{abstract}

Keywords: Nanocomposite, organophilization, non-ionic surfactant, purified clay. 


\section{Introdução}

Os nanocompósitos poliméricos oferecem uma série de vantagens significativas sobre os compósitos convencionais. Enquanto os compósitos convencionais requerem entre $10-50 \%$ em peso de cargas a fim de conferir propriedades mecânicas e térmicas, satisfatórias, os nanocompósitos têm propriedades similares ou superiores com teores de carga inferiores a $5 \%$ em peso e com a vantagem de possuir menor densidade e sem comprometer a processabilidade ${ }^{[1]}$.

Um dos métodos mais empregados na obtenção de nanocompósitos é o da intercalação por fusão, pois é possível a obtenção de nanocompósitos poliméricos por meio dos processos convencionais (extrusão e injeção) sem a utilização de solventes, reduzindo o numero de etapas, riscos ambientais e custo ${ }^{[2-4]}$.

Dentre as cargas mais utilizadas no desenvolvimento destes materiais encontram-se as argilas bentoníticas. Estas argilas podem ser definidas como argilas constituídas essencialmente de argilominerais do grupo da esmectita, especialmente a montmorilonita. São fatores que controlam as propriedades das argilas: a composição mineralógica dos argilominerais e nãoargilominerais existentes, suas respectivas distribuições granulométricas, teor de eletrólitos, cátions trocáveis, sais solúveis, natureza e teor de componentes orgânicos e características texturais ${ }^{[5,6]}$.

Elementos contaminantes são amplamente encontrados nas variedades nacionais das argilas bentoníticas, geralmente na forma de sais inorgânicos, minerais acessórios como quartzo, pirita, mica, caulinita, etc. Estes contaminantes podem, no caso dos usos já mencionados, prejudicar e mesmo inviabilizar seu uso industrial, com especial destaque para os nanocompósitos poliméricos ${ }^{[7-9]}$. Para retirada dos contaminantes na forma de acessórios das argilas naturais foi utilizado por Martins ${ }^{[10]}$ um sistema de hidrociclonagem, em circuito fechado, visando sua purificação. $\mathrm{O}$ autor concluiu que a purificação pelo hidrociclone se mostrou eficaz para retirada das frações grosseiras das argilas.

Para melhorar a interação com o polímero as argilas bentoníticas precisam ser modificadas organicamente com uso de tensoativos. Atualmente o uso de tensoativos não iônicos vem paulatinamente sendo utilizados, e, diferentemente dos tensoativos iônicos o principal mecanismo de modificação da argila é governado por fenômenos de adsorção, principalmente na camada tetraédrica do argilomineral pertencente ao grupo das esmectítas ou bentonitas, conforme demonstrados nos trabalhos de Shen ${ }^{[11]}$ e Yuang et al. ${ }^{[12]}$.

Dentre os polímeros empregados como matriz na preparação de nanocompósitos polímero/argila, o polipropileno (PP) é um dos mais usados, pois exibe uma atrativa combinação de baixo custo e grande versatilidade em termos de propriedades, aplicações e reciclagem ${ }^{[13]}$. Contudo, este polímero não possui nenhum grupo polar em sua cadeia que possa interagir com grupos polares presentes nas superfícies das argilas usadas como nano cargas. A ausência de polaridade no PP dificulta a interação e intercalação das cadeias poliméricas entre as lamelas das partículas de argila. Porém para promover interações, principalmente, por meio de pontes de hidrogênio, com as hidroxilas presentes na superfície da argila e facilitar o processo de intercalação, utilizam-se agentes compatibilizantes ${ }^{[13-15]}$.

Na literatura não há, até o presente, trabalhos sobre o desenvolvimento de nanocompósitos com matriz polimérica onde a argila foi modificada em escala piloto com tensoativo não iônico. Além disso, as argilas utilizadas neste trabalho são da região de Cubati, estado da Paraíba - PB. Portanto, o objetivo principal deste trabalho é a purificação e organofilização em escala piloto de argilas bentoníticas com tensoativo não iônico e a influência destas argilas nas propriedades mecânicas e térmicas de nanocompósitos de PP/compatibilizante/ argila.

\section{Experimental}

\section{Materiais}

$\mathrm{Na}$ preparação dos compósitos, empregou-se como matriz um Polipropileno (PP), homopolímero, código H103, em grânulos, $\mathrm{IF}=40 \mathrm{~g} / 10 \mathrm{~min} \quad(2,16$ $\mathrm{kg} / 230^{\circ} \mathrm{C}$ - ASTM D-1238-L), fabricado pela Braskem S.A. Como carga utilizou-se argilas bentoníticas nacionais (Argila Cinza e Argila Verde) provenientes de Cubati - PB. Na organofilização destas argilas foi utilizado um tensoativo não-iônico Ultramina TA50®, que é uma Amina Graxa de Sebo $5 \mathrm{EO}$, onde $\mathrm{EO}=$ número médio de moles de óxido de etileno, gentilmente cedido pelo fabricante Oxiteno. O compatibilizante utilizado para melhorar a afinidade entre a matriz polimérica e a superfície das argilas foi um copolímero de etilenometacrilato de glicidila (E-GMA), Lotader® AX 8840 fornecido pela Arkema. Densidade de $0,94 \mathrm{~g} / \mathrm{cm}^{3}$, $\mathrm{IF}=5 \mathrm{~g} / 10 \mathrm{~min}\left(190^{\circ} \mathrm{C} / 2,16 \mathrm{~kg}\right)$. Contém $8 \%$ de metacrilato glicidila. As estruturas dos materiais utilizados neste trabalho encontram-se na Tabela 1 .

\section{Tratamento da argila: purificação e organofilização}

\section{Purificação com hidrociclone}

As argilas cinza e verde naturais $(\mathrm{CN}$ e $\mathrm{VN})$ passaram por um processo de secagem e desagregação. Em seguida foram dispersas numa concentração de $4 \%$ em massa, num volume de $30 \mathrm{~L}$ de água destilada mantida sob agitação por um período de $24 \mathrm{~h}$ em temperatura ambiente. Após esse período, a dispersão foi bombeada com o auxilio de uma bomba centrífuga para o hidrociclone onde ocorre o processo de separação do fluxo em "overflow" e "underflow". O "underflow" é colhido na parte inferior, ápex, (rejeito), e o "overflow" na parte superior, vórtex, (purificado).

Os orifícios utilizados, o vórtex e o ápex, podem ser variados, essa variação resulta na modificação da concentração e da vazão. As possibilidades podem ser vistas na Tabela 2 e dentre elas foi selecionada a melhor configuração do hidrociclone, no caso a A1, a qual foi mais eficaz no processo de purificação das argilas, conforme observado por Costa et al. ${ }^{[16]}$.

As argilas cinza e verde purificadas foram denominadas $\mathrm{CP}$ e VP respectivamente. 
Processo de organofilização em escala piloto

O processo de organofilização das argilas purificadas foi realizado em escala piloto segundo o procedimento descrito a seguir: as dispersões de argila em água destilada foram preparadas com velocidade de agitação de $1750 \mathrm{rpm}$, adicionando-se tensoativo não iônico Ultramina ${ }^{\circledR}$ TA50, no teor de $30 \%$, em relação ao teor de argila seca, mantendo a agitação por $20 \min ^{[17]}$. Após esse tempo, o material obtido foi passado pelo filtro prensa e os aglomerados obtidos foram secados em estufa a $60^{\circ} \mathrm{C}$, desagregados em um almofariz elétrico, passados em peneira $A B N T n^{\circ} 200(D=74 \mu \mathrm{m})$ e caracterizados.

As argilas $\mathrm{CP}$ e VP organofilizadas com o tensoativo não iônico foram denominadas de CPO e VPO respectivamente.

\section{Preparação dos compósitos}

A argila modificada foi secada em estufa na temperatura de $60^{\circ} \mathrm{C}$ durante 24 horas e, em seguida, misturada em concentração de 1:1 com o compatibilizante E-GMA em misturador interno Rheomix 600 acoplado a um reômetro de torque da Haake Buchcler na temperatura de $200^{\circ} \mathrm{C}$ e velocidade dos rotores de $60 \mathrm{rpm}$, durante 10 minutos. $\mathrm{O}$ concentrado resultante foi posteriormente, triturado e misturado com o polipropileno (PP H103) em concentração de $3 \%$ em peso de argila.

Tabela 1. Estrutura dos Materiais utilizados neste trabalho.

\begin{tabular}{|c|c|}
\hline Material & Estrutura \\
\hline PP & $\left\{\left.\begin{array}{r}\mathrm{CH}_{2}-\mathrm{CH} \\
\mathrm{CH}_{3}\end{array}\right|_{\mathrm{n}}\right.$ \\
\hline E-GMA & $-\left(\mathrm{CH}_{2}-\mathrm{CH}_{2}\right)_{\mathrm{x}}-\left(\mathrm{CH}_{2}-\stackrel{\left.\left.\right|_{\mid} ^{\mathrm{C}}\right)}{\mathrm{CH}_{3}}-\right.$ \\
\hline $\begin{array}{l}\text { Tensoativo } \\
\text { Ultramina }{ }^{\circledR} \\
\text { TA } 50\end{array}$ & $\begin{array}{l}\text { Onde } \mathrm{n}=\text { grau de etoxilação } \\
\mathrm{R}=\mathrm{H}_{3} \mathrm{C}\left(\mathrm{CH}_{2}\right)_{17} \text { cadeia carbônica média da amina } \\
\text { graxa }\end{array}$ \\
\hline
\end{tabular}

Tabela 2. Dimensões dos orifícios utilizados.

\begin{tabular}{|c|c|c|c|c|}
\hline \multicolumn{2}{|c|}{$\begin{array}{c}\text { Vórtex } \\
\text { (Diâmetro interno) }\end{array}$} & \multicolumn{3}{|c|}{$\begin{array}{c}\text { Ápex } \\
\text { (Diâmetro interno) }\end{array}$} \\
\hline A & B & 1 & 2 & 3 \\
\hline $5 \mathrm{~mm}$ & $6 \mathrm{~mm}$ & $4 \mathrm{~mm}$ & $3 \mathrm{~mm}$ & $5 \mathrm{~mm}$ \\
\hline
\end{tabular}

Em seguida o PP misturado ao concentrado foi alimentado em extrusora de dupla-rosca, corrotacional modular, modelo ZSK de $18 \mathrm{~mm}$ da Coperion-WernerPfleiderer. A taxa de alimentação foi de $5 \mathrm{Kg} / \mathrm{h}$, perfil de temperatura utilizado foi de $200^{\circ} \mathrm{C}$ para todas ao zonas, com velocidade de rosca de $250 \mathrm{rpm}$. A configuração de rosca utilizada foi com elementos de mistura dispersiva e distributiva, conforme Figura 1.

Após extrudados, os materiais foram secados em estufa sob vácuo a $80^{\circ} \mathrm{C}$ por $12 \mathrm{~h}$. As amostras para os ensaios de tração e impacto foram moldadas por injeção, segundo as normas ASTM D638 e D256, respectivamente. Foi utilizada uma injetora Fluidmec, modelo H3040. A temperatura de moldagem foi de $200^{\circ} \mathrm{C}$ e a do molde de $20^{\circ} \mathrm{C}$. As composições das amostras encontram-se na Tabela 3 .

\section{Caracterização das argilas e dos compósitos}

\section{Análise granulométrica por difração de laser (AG)}

A análise granulométrica por difração de laser foi feita em uma dispersão da argila bentonítica colocada em um equipamento CILAS modelo 1064, em modo úmido, até atingir a concentração ideal que é de 170 unidades de difração/área de incidência. Foi utilizada para avaliar a purificação de argila.

\section{Análises Químicas (AQ)}

As análises químicas por fluorescência de raios $\mathrm{X}$, EDX, foram efetuadas em equipamento EDX 720 da Shimadzu, com as amostras de argila passadas em peneira ABNT n 200 (0,074mm). Foi utilizada em conjunto com a AG para avaliar o efeito da purificação da argila.

\section{Difração de raios X (DRX)}

A análise por difração de raios $\mathrm{X}$ (DRX), das argilas e dos compósitos foi realizada utilizando-se um equipamento modelo XRD-6000 da Shimadzu. A radiação utilizada foi $\mathrm{K} \alpha$ do $\mathrm{Cu}(40 \mathrm{kV} / 30 \mathrm{~mA})$; a velocidade do goniômetro foi de $2 \%$ min e passo de $0,02^{\circ}$ e com $2 \theta$ variando de 2 até $60^{\circ}$ para avaliar a purificação da argila. Para avaliar tanto o grau de intercalação do tensoativo não iônico nas argilas como também o grau de dispersão (intercalação/esfoliação) das argilas no polímero, o $2 \theta$ foi variado entre 2 e $10^{\circ}$. Para avaliar a influência das argilas na cristalinidade do PP foi utilizado o $2 \theta$ variando de 10 até $35^{\circ}$.

\section{Espectroscopia no infravermelho (IV)}

As análises no IV das argilas foram realizadas em um espectrômetro de infravermelho de marca Perkin Elmer Spectrum 400 no modo de Refletância Total Atenuada (ATR) e com varredura de 4000 a $650 \mathrm{~cm}^{-1}$. As amostras foram caracterizadas na forma de pó. Foram realizadas no

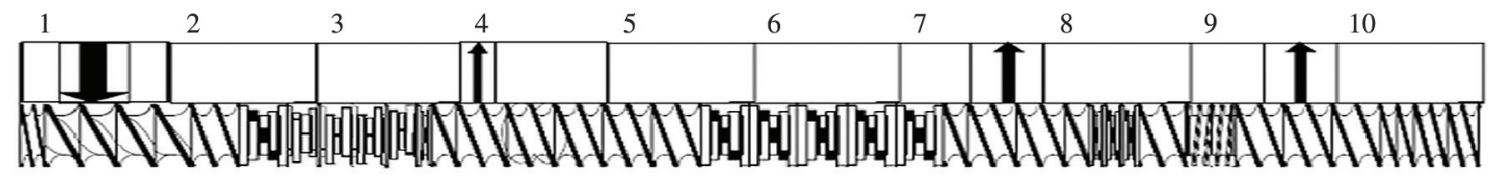

Figura 1. Perfil de rosca utilizado na extrusora dupla-rosca corrotacional. 
Tabela 3. Composição das amostras.

\begin{tabular}{lcccc}
\hline \multicolumn{1}{r}{ Amostras } & PP $(\%)$ & E-GMA $(\%)$ & CPO (pcr) & VPO (pcr) \\
\hline PP & 100 & ---- & ---- & $-\cdots$ \\
PP/E-GMA/CPO & 90 & 10 & 03 & --- \\
PP/E-GMA/VPO & 90 & 10 & ---- & 03 \\
\hline
\end{tabular}

Tabela 4. Análise granulométrica por difração de laser (AG) das argilas de Cubati, PB.

\begin{tabular}{ccccc}
\hline Amostra & Diâmetro médio $(\boldsymbol{\mu m})$ & Diâmetro a 50\% $(\boldsymbol{\mu m})$ & $\begin{array}{c}\text { Volume acumulado } \\
\text { com } \Phi \text { médio abaixo } \\
\text { de } \mathbf{2} \boldsymbol{\mu m}(\boldsymbol{\%})\end{array}$ & $\begin{array}{c}\text { Maior concentração de } \\
\text { partículas }(\boldsymbol{\mu m})\end{array}$ \\
\hline $\mathrm{CN}$ & 7,87 & 16,47 & $4-8$ \\
$\mathrm{CP}$ & 5,43 & 5,00 & 15,51 & $4-8$ \\
$\mathrm{VN}$ & 8,24 & 5,61 & 17,13 & $4-8$ \\
$\mathrm{VP}$ & 4,86 & 3,29 & 20,45 & $4-8$ \\
\hline
\end{tabular}

CN: Cinza Natural, CP: Cinza Purificada, VN: Verde Natural, VP: Verde Purificada.

mínimo duas determinações para cada composição. Esta técnica foi utilizada em conjunto com a DRX para avaliar se houve intercalação do tensoativo nas argilas.

\section{Análise Termogravimétrica (TG)}

As análises foram conduzidas em aparelho TGA 50 da Shimadzu na AEMa/UFCG/Laboratório de Reciclagem e Resíduos Sólidos, com faixa de temperatura que variou da temperatura ambiente a $800^{\circ} \mathrm{C}$, taxa de aquecimento $12,5^{\circ} \mathrm{C} / \mathrm{min}$, utilizando atmosfera de nitrogênio e um porta amostra de platina.

\section{Calorimetria Exploratória Diferencial (DSC)}

A influência da argila na temperatura de fusão do PP foi avaliada por DSC em equipamento TA Instruments , modelo Q20. As condições de teste foram: fluxo de $\mathrm{N}_{2}$ de $100 \mathrm{ml} / \mathrm{min}$; aquecimento de $30^{\circ} \mathrm{C}$ a $220^{\circ} \mathrm{C}$ a $10^{\circ} \mathrm{C} / \mathrm{min}$; resfriamento $220^{\circ} \mathrm{C}$ a $30^{\circ} \mathrm{C}$ a $10^{\circ} / \mathrm{min}$.

\section{Ensaio mecânico de tração}

O ensaio de tração foi realizado segundo a norma ASTM D 638. Os ensaios foram conduzidos em máquina universal de ensaios AG-IS $100 \mathrm{kN}$ da Shimadzu, empregando-se uma velocidade de deformação de 50 $\mathrm{mm} / \mathrm{min}$.

\section{Ensaio mecânico de impacto}

Os ensaios de resistência ao impacto IZOD foram realizados em equipamento do modelo RESIL 5,5 da CEAST e pêndulo de $2,75 \mathrm{~J}$, de acordo com a norma ASTM D 256. Os entalhes de 2,5 mm foram feitos em um entalhador NOTSCHVIS da CEAST.

\section{Resultados e Discussão}

\section{Caracterização das argilas}

\section{Análise granulométrica por difração de laser (AG)}

As Tabelas 4 e 5 apresentam os resultados das análises granulométricas das argilas cinza e verde naturais $(\mathrm{CN}$ e VN) e purificadas (CP e VP). Observa-se que com a purificação, a argila cinza apresentou uma redução de $31 \%$ no diâmetro médio das suas partículas (de 7,87 para $5,43 \mu \mathrm{m}$ ). Já para o diâmetro a $50 \%$, a redução foi de $8 \%$ (de 5,00 para $4,61 \mu \mathrm{m}$ ). A maior concentração de
Tabela 5. Distribuição granulométrica por tamanho de partículas das argilas cinza e verde naturais $(\mathrm{CN}$ e $\mathrm{VN})$ e purificadas $(\mathrm{CP}$ e VP).

\begin{tabular}{cccc}
\hline Amostra & $\begin{array}{c}\text { Argila }(\boldsymbol{\%}) \\
(\mathbf{x}<\mathbf{2} \boldsymbol{\mu m})\end{array}$ & $\begin{array}{c}\text { Silte }(\boldsymbol{\%}) \\
(\mathbf{2} \boldsymbol{\mu} \mathbf{m}<\mathbf{x}<\mathbf{2 0} \boldsymbol{\mu m})\end{array}$ & $\begin{array}{c}\text { Areia }(\boldsymbol{\%}) \\
(\mathbf{x}>\mathbf{2 0} \boldsymbol{\mu} \mathbf{m})\end{array}$ \\
\hline CN & 16,47 & 74,09 & 9,44 \\
CP & 15,51 & 84,28 & 0,21 \\
VN & 17,13 & 73,02 & 9,85 \\
VP & 20,45 & 79,33 & 0,22 \\
\hline
\end{tabular}

partículas permaneceu com diâmetro entre 4 e $8 \mu \mathrm{m}$ e o volume acumulado com diâmetro médio abaixo de $2 \mu \mathrm{m}$ sofreu um decréscimo de $5 \%$, que corresponde à fração argila. O teor de silte aumentou em $14 \%$ enquanto que o teor de areia foi significativamente reduzido em $98 \%$ (de 9,44 para $0,21 \%$ ).

Analisando a argila verde, observa-se que houve uma redução de $41 \%$ no diâmetro médio das partículas e de $25 \%$ no diâmetro a $50 \%$. A maior concentração de partículas permaneceu com diâmetro entre 4 e $8 \mu \mathrm{m}$ e houve um acréscimo de $19 \%$ no volume acumulado com diâmetro médio abaixo de $2 \mu \mathrm{m}$, que corresponde à fração argila. O teor de silte aumentou $9 \%$ e houve uma redução sigificativa (de $\sim 98 \%$ ) no teor de areia.

Dessa forma, observa-se claramente a redução do tamanho médio equivalente das partículas após o processo de hidrociclonagem, uma diminuição significativa no teor de areia, indicando que houve a purificação efetiva da argila.

\section{Composição química por fluorescência de raios X (EDX)}

Na Tabela 6 estão apresentados os resultados da composição química das argilas natural $(\mathrm{CN}$ e $\mathrm{VN})$ e purificada (CP e VP).

A redução no teor de sílica com a purificação principalmente na argila verde purificada (VP) se deve provavelmente a eliminação de sílica livre na forma de grão de quartzo após o processo de purificação ${ }^{[18]}$. Após o processo de purificação, o teor de $\mathrm{SiO}_{2}$ presente na composição das argilas foi maior para a argila CP. A argila VP apresentou teor de $\mathrm{Fe}_{2} \mathrm{O}_{3}$ superior ao da argila CP. Estes teores de $\mathrm{Fe}_{2} \mathrm{O}_{3}$ são provavelmente dos argilominerais do grupo da esmectita, ou seja, montmorilonita, segundo 
dados de Souza Santos ${ }^{[6]}$, sendo também semelhantes aos teores observados nas amostras de Boa Vista, $\mathrm{PB}^{[19-21]}$. Verifica-se que as amostras apresentaram perda ao fogo entre 10 e $16 \%$, o que está relacionado, provavelmente, à perda de águas coordenadas e adsorvidas, hidroxilas dos argilominerais e queima da matéria orgânica. Analisando os resultados da Tabela 6 observa-se que as argilas possuem composição química típica das argilas bentoníticas ${ }^{[5,6,21]}$. Os resultados da análise química em conjunto com os resultados da análise granulométrica por difração de laser confirmam que as argilas foram purificadas com sucesso.

\section{Difração de raios $X(D R X)$}

A Figura 2 apresenta os resultados de difração de raios $\mathrm{X}$ das argilas cinza e verde naturais $(\mathrm{CN}$ e $\mathrm{VN})$ e purificadas (CP e VP).

Analisando os difratogramas observa-se que tanto as argilas naturais ( $\mathrm{CN}$ e VN) e argilas purificadas (CP e VP) possuem argilomineral esmectítico, caulinita e quartzo como fases cristalinas; entretanto, ocorreu uma redução significativa nas intensidades dos picos associados ao quartzo, o que indica que o tratamento de purificação foi eficiente na remoção de grande parte do quartzo presente nas argilas, corroborando os resultados da análise granulométrica.

A Figura 3 ilustra os DRX das argilas cinza e verde purificadas (CP e VP) e purificadas e organofilizadas (CPO e VPO). Figura 3a ilustra os difratogramas das argilas cinza purificada (CP) e cinza purificada e organofilizada (CPO). Observa-se que a argila $\mathrm{CP}$ apresentou um pico característico do argilomineral esmectítico com espaçamento basal $\mathrm{d}_{001}$ de $15,0 \AA$ A. A argila CPO apresentou dois picos de difração em $2 \theta$ de 2 e $5,18^{\circ}$ que correspondem ao espaçamento basal de 44,1 e $17,0 \AA$, respectivamente. O resultado $\left(2 \theta \sim 2^{\circ}\right)$ confirma a intercalação do tensoativo não iônico entre as lamelas da argila organofílica. O pico em $2 \sim 5,18^{\circ}$ está possivelmente relacionado à alguma quantidade de

Tabela 6. Composição química das argilas de Cubati, PB, após hidrociclonagem.

\begin{tabular}{|c|c|c|c|c|c|c|c|c|}
\hline Amostra & $\mathrm{SiO}_{2}(\%)$ & $\mathrm{Al}_{2} \mathrm{O}_{3}(\%)$ & $\mathrm{Fe}_{2} \mathrm{O}_{3}(\%)$ & $\mathrm{CaO}(\%)$ & $\operatorname{MgO}(\%)$ & $\mathrm{K}_{2} \mathrm{O}(\%)$ & $\begin{array}{c}\text { Outros } \\
\text { Óxidos }(\%)\end{array}$ & PF (\%) \\
\hline $\mathrm{CN}$ & 53,495 & 26,125 & 3,655 & 0,797 & 3,240 & 0,841 & 1,257 & 10,588 \\
\hline $\mathrm{CP}$ & 52,872 & 26,140 & 3,685 & 0,786 & 3,218 & 0,644 & 1,132 & 11,450 \\
\hline $\mathrm{VN}$ & 50,429 & 22,609 & 10,234 & 0,568 & 2,838 & 1,666 & 1,204 & 10,451 \\
\hline VP & 45,831 & 21,461 & 10,371 & 0,657 & 2,693 & 1,428 & 1,147 & 16,410 \\
\hline
\end{tabular}

PF: Perda ao Fogo CN: Cinza Natural, CP: Cinza Purificada, VN: Verde Natural, VP: Verde Purificada.

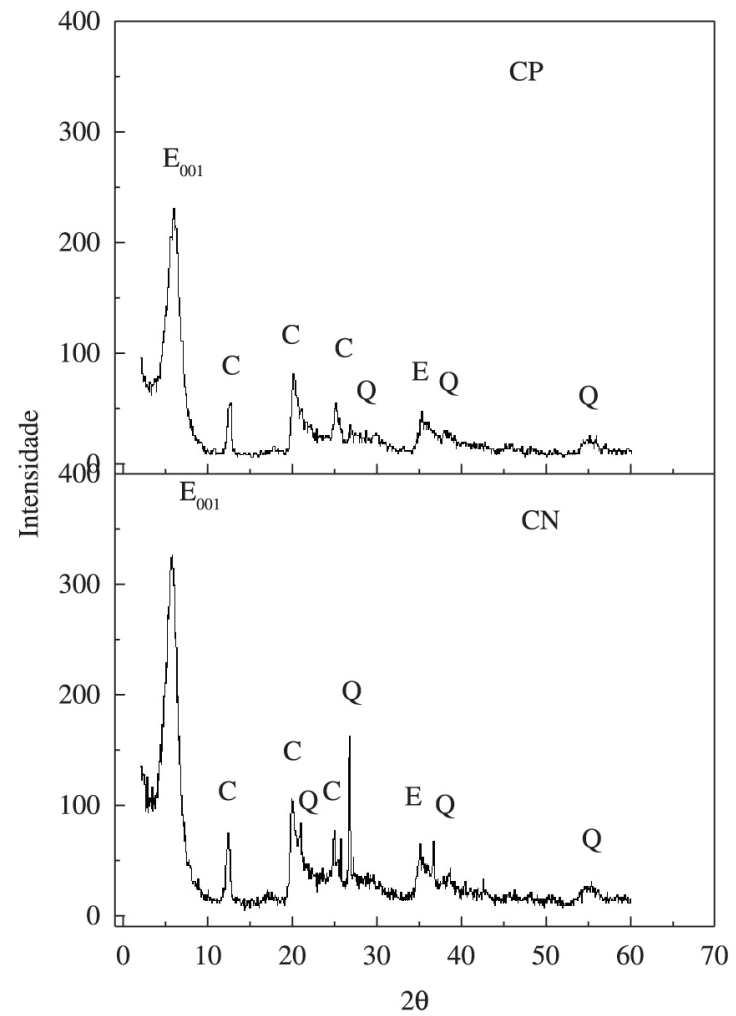

(a)

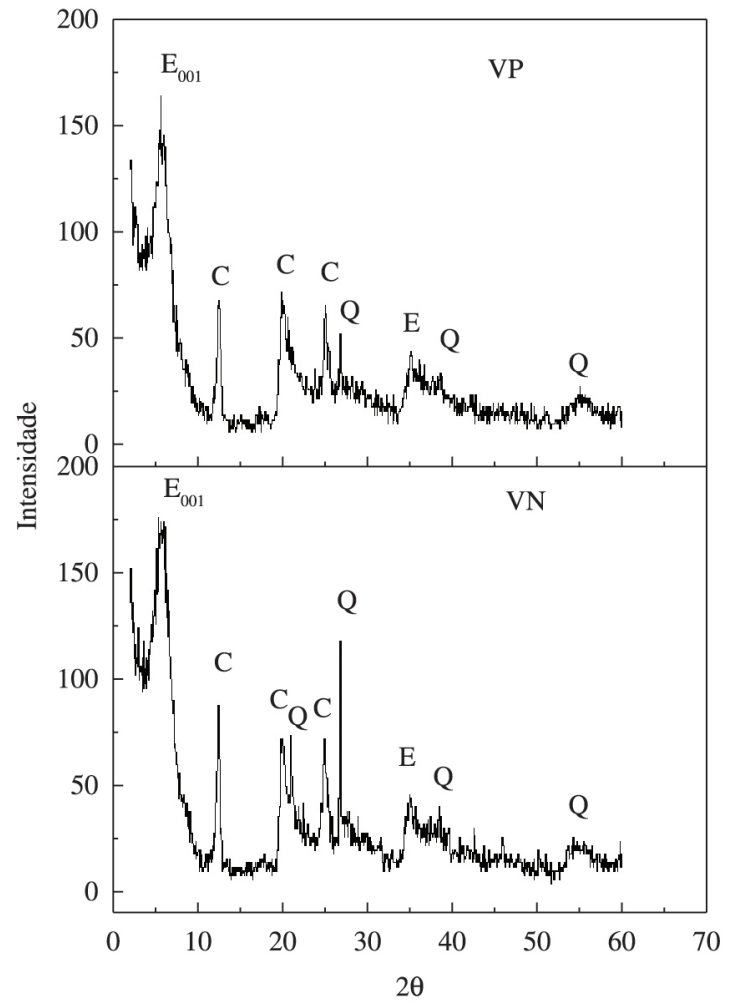

(b)

Figura 2. Difratogramas de raio $\mathrm{X}$ das argilas naturais $(\mathrm{CN}$ e $\mathrm{VN}$ ) e purificadas (CP e VP). 


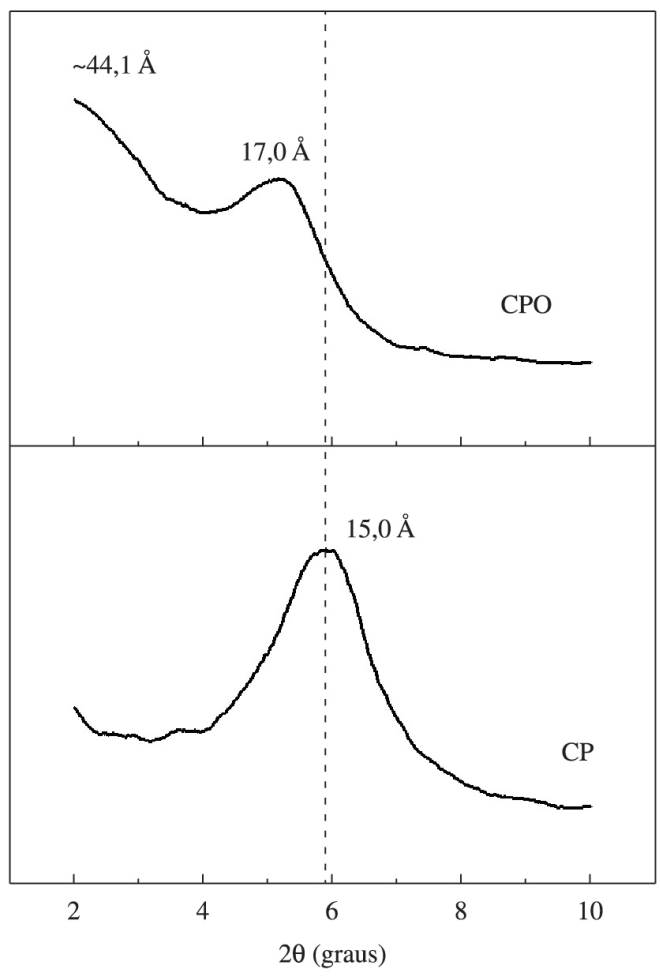

(a)

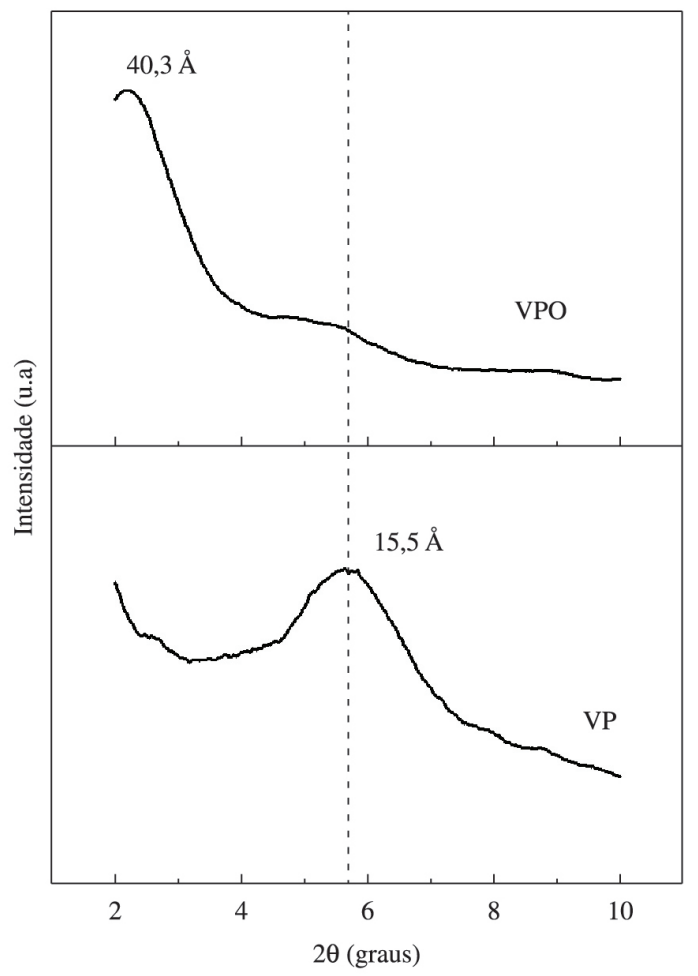

(b)

Figura 3. DRX das argilas purificadas e purificadas e organofilizadas: a) CP e CPO; b) VP e VPO.

argila não intercalada permanecendo numa posição muito próxima à da argila $\mathrm{CP}\left(2 \theta \sim 5,87^{\circ}\right)$.

A Figura $3 b$ ilustra os difratogramas de Raios-X das argilas verde purificada (VP) e verde purificada e organofílizada (VPO).

Observa-se que o difratograma da argila VP apresentou um pico em $2 \theta \sim 5,70^{\circ}$ correspondendo ao $\mathrm{d}_{001}$ de $15,5 \AA$ e quando organofilizada (VPO) o pico foi deslocado para $2 \theta \sim 2,19^{\circ}$ correspondendo a um espaçamento basal $d_{001}$ de 40,3 $\AA$. Observa-se que a distância interplanar original da argila teve um aumento considerável com a incorporação do tensoativo, o que significa que houve intercalação do tensoativo não iônico entre as lamelas da argila. Comparando-se as duas argilas, é possível observar que o aumento na distância interplanar basal $\left(\mathrm{d}_{001}\right)$, referente ao plano de reflexão (001) com a adição do tensoativo foi maior para a argila CP. Este aumento maior para a argila $\mathrm{CP}$ pode ser atribuído ao maior teor de $\mathrm{SiO}_{2}$ presente na composição desta argila. De acordo com a literatura ${ }^{[11,12]}$, as cadeias de óxido de etileno (EO) presentes no tensoativo não iônico, são fortemente adsorvidas no $\mathrm{SiO}_{2}$ presente na argila via formação de pontes de hidrogênio na presença de água, e consequentemente levando à expansão da argila. Isto explica o maior aumento do espaçamento basal $\mathrm{d}_{001}$ para a argila CP, pois a mesma possui um teor mais elevado de $\mathrm{SiO}_{2}$ do que a argila VP.

\section{Espectroscopia no infravermelho (IV)}

$\mathrm{Na}$ Figura 4 estão ilustrados os espectros no infravermelho das argilas cinza purificada (CP) e cinza purificada e organofilizada (CPO).

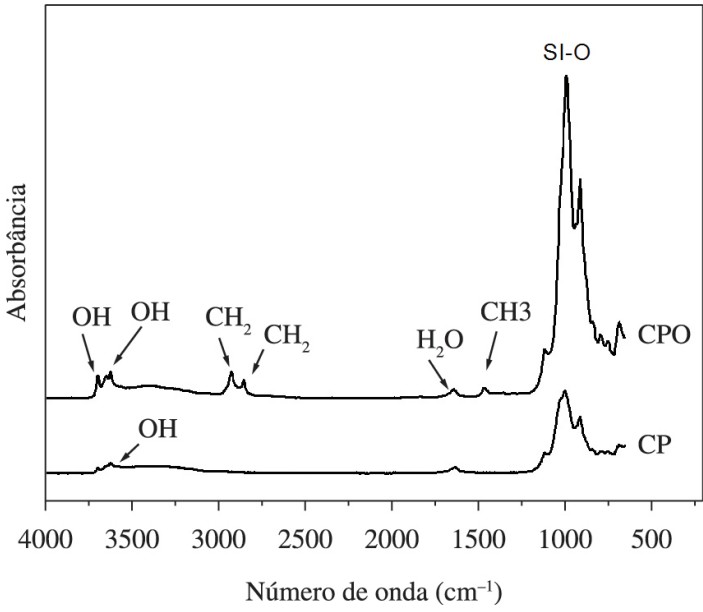

Figura 4. Espectros no infravermelho das argilas CP e CPO.

A argila CPO apresenta absorção a 3692 e 3616 cm$^{-1}$, característica da presença de hidroxilas; observam-se também absorções entre 2920 e $2854 \mathrm{~cm}^{-1}$, referente às vibrações de deformação axial assimétrica dos grupos $\mathrm{CH}_{2}$ e $\mathrm{CH}_{3}$ presentes na estrutura na cadeia carbônica da amina graxa que faz parte da estrutura química do tensoativo (Tabela 1) a $1644 \mathrm{~cm}^{-1}$, características de água adsorvida; de 1351 a $1464 \mathrm{~cm}^{-1}$ referentes à deformação angular assimétrica e simétrica de grupos $\mathrm{CH}_{3}$, também presentes na estrutura do tensoativo, o que demonstra a presença do tensoativo não iônico na argila. O pico a $991 \mathrm{~cm}^{-1}$ é característico das ligações Si-O-Si e nas faixas 
de $903 \mathrm{~cm}^{-1}, 783 \mathrm{~cm}^{-1}$ e $678 \mathrm{~cm}^{-1}$, observaram-se os picos característicos das folhas octaédricas.

Na Figura 5 está ilustrado o espectro na região de infravermelho das argilas verde purificada (VP) e verde purificada e organofilizada (VPO).

A argila VPO apresenta absorção a 3700 e $3627 \mathrm{~cm}^{-1}$, características da presença de hidroxilas; observa-se também absorções entre 2914 e 2851 cm$^{-1}$, referentes às vibrações de deformação axial assimétrica do grupo $\mathrm{CH}_{2}$ a $1644 \mathrm{~cm}^{-1}$, características de água adsorvida; de 1239 a

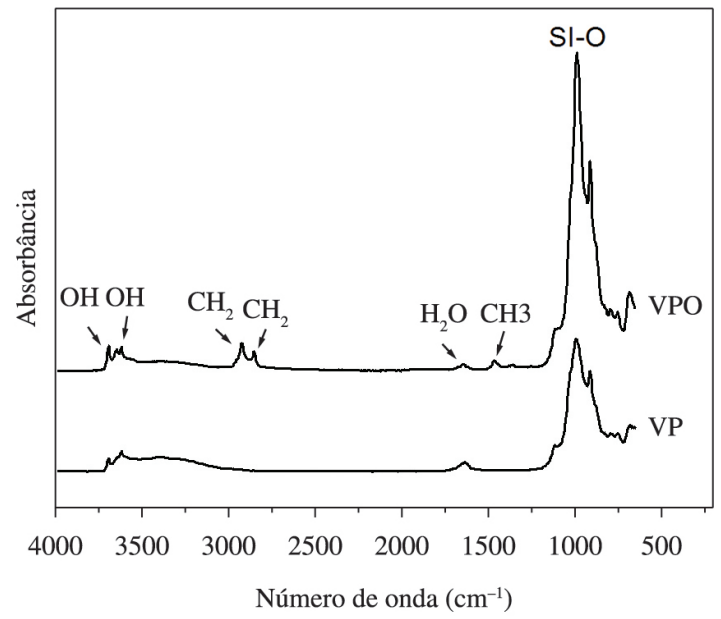

Figura 5. Espectros no infravermelho das argilas VP e VPO.

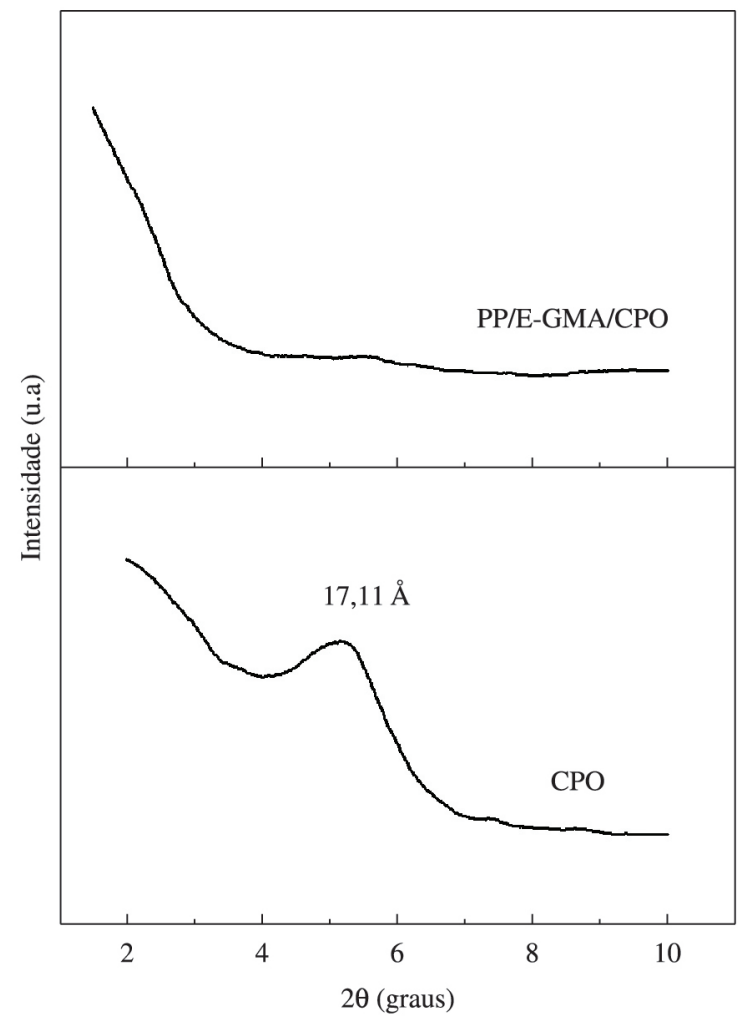

(a)
$1456 \mathrm{~cm}^{-1}$ referentes à deformação angular assimétrica e simétrica de grupos $\mathrm{CH}_{3}$. Como observado anteriormente, tanto os grupos $\mathrm{CH}_{3}$ quanto os grupos $\mathrm{CH}_{2}$ fazem parte da estrutura química do tensoativo (Tabela 1), o que demonstra a presença do tensoativo não iônico na argila; a $983 \mathrm{~cm}^{-1}$, característica das ligações Si-O-Si e nas faixas de $902 \mathrm{~cm}^{-1}, 807 \mathrm{~cm}^{-1}$ e $678 \mathrm{~cm}^{-1}$, observaram-se os picos característicos das folhas octaédricas.

\section{Caracterização dos compósitos}

\section{Difração de Raios-X}

A Figura 6a, b ilustra os difratogramas de DRX das argilas organofílicas e dos sistemas PP/E-GMA/CPO e PP/E-GMA/VPO. Na Figura 6a é possível observar no sistema PP/E-GMA/CPO que houve o desaparecimento do segundo pico de difração $\left(2 \theta \sim 5,18^{\circ}\right)$ observado na argila organofílica com espaçamento basal de 17,0 enquanto que o primeiro pico $\left(2 \theta \sim 2^{\circ}\right)$ foi deslocado para ângulo $2 \theta$ menor, indicando que houve uma boa dispersão da argila no polímero, e que para este sistema foi obtido um nanocompósito com estrutura intercalada. Já para o sistema PP/E-GMA/VPO (Figura 6b) é possível observar que não houve o deslocamento do pico (001) para ângulo $2 \theta$ menor, isto é, o espaçamento basal $\left(\mathrm{d}_{001}\right)$ da argila modificada neste sistema foi semelhante ao da argila organofílica, indicando que houve a formação de um microcompósito quando comparada com a CPO. Provavelmente, a argila VPO por ter expandido menos, conforme observado no DRX da Figura 3, dificultou a

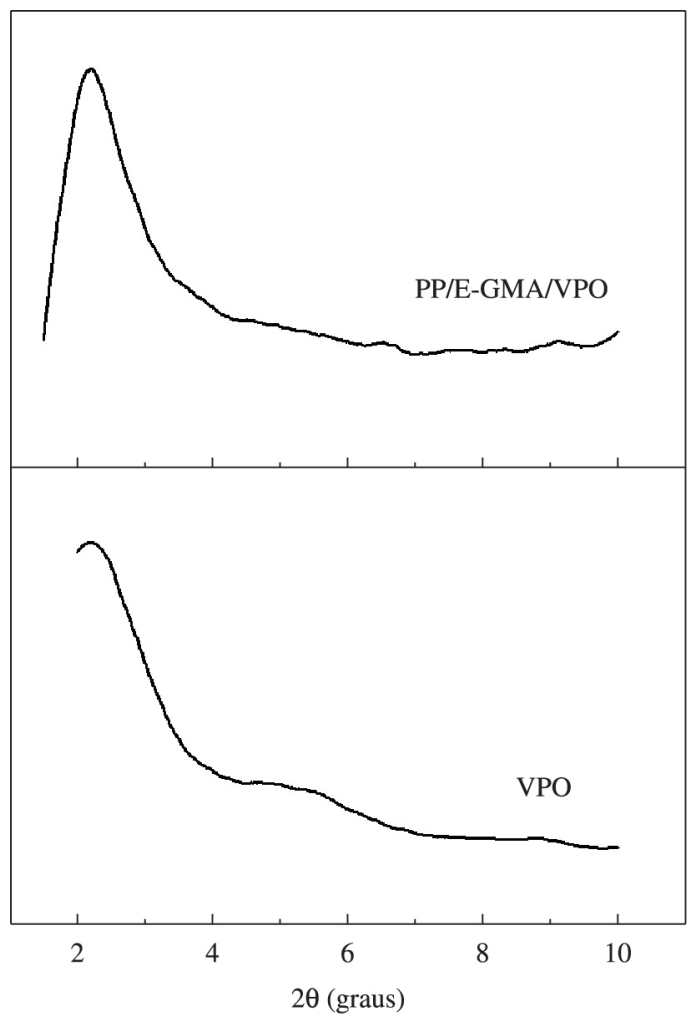

(b)

Figura 6. Difratogramas de raios $\mathrm{X}$ das argilas organofílicas e dos sistemas PP/E-GMA/argila. 
inercalação do polímero entre as galerias, resultando na formação de um microcompósito. Conforme discutido anteriormente, a argila VP, por possuir menor teor de $\mathrm{SiO}_{2}$, apresenta baixa adsorção de cadeias de óxido de etileno (EO) presentes no tensoativo não iônico.

\section{Influência das argilas na estrutura cristalina do polipropileno}

Para avaliar o efeito das argilas CPO e VPO na estrutura cristalina do Polipropileno (PP), o PP e os sistemas PP/E-GMA/argila foram analisados por DRX. A Figura 7 ilustra os difratogramas de raios-X dos sistemas (PP/E-GMA/CPO e PP/E-GMA/VPO), com $2 \theta$ variando de 10 a $35^{\circ}$. Tanto o PP puro quanto os sistemas estudados apresentaram fase cristalina monoclínica, mostrando reflexões de Bragg observados nos difratogramas correspondendo aos planos (110), (040), (130), (111), (131), respectivamente.

Os picos de difração característicos do $\mathrm{PP}$ permaneceram praticamente inalterados na faixa $2 \theta=10$ a $35^{\circ}$ para os sistemas preparados, indicando que eles também contêm apenas a fase cristalina $\alpha$. Segundo a literatura ${ }^{[14,22-29]}$, estudos com nanocompósitos de PP/argila organofílica, mostraram que a presença de argila modificada, independente do tensoativo e do compatibilizante utilizado, não altera a natureza cristalográfica do PP.

\section{Calorimetria Exploratória Diferencial (DSC)}

Na Figura 8 estão apresentadas as curvas de DSC obtidas a partir de corpos de prova do PP puro e dos sistemas PP/E-GMA/CPO e PP/E-GMA/VPO.

Observa-se que não houve diferença significativa entre as curvas apresentadas pelos sistemas PP/E-GMA/ argila, evidenciando que a temperatura de fusão do PP não foi alterada pela presença da argila e pelo tipo de argila bentonita (cinza ou verde) utilizada neste trabalho. Outros autores ${ }^{[29,30]}$, mesmo utilizando tensoativos iônicos e compatibilizantes diferentes, também observaram que não houve variação significativa na temperatura de fusão $\left(\mathrm{T}_{\mathrm{m}}\right)$ dos compósitos de PP/argila quando comparados ao PP.

\section{Análise termogravimétrica (TG/DTG)}

A Figura 9 ilustra o gráfico de análise termogravimétrica do PP puro e dos sistemas PP/EGMA/CPO e PP/E-GMA/VPO. Observa-se que a incorporação das argilas organofilizadas CPO e VPO ao PP teve influência na estabilidade térmica do polímero. Entre os sistemas estudados o que apresentou maior estabilidade térmica foi aquele contendo a argila CPO (PP/E-GMA/CPO). Isto pode ser atribuído à melhor dispersão da argila neste sistema (conforme observado na Figura 6), dificultando a difusão de oxigênio para o interior do material. Ding et al. ${ }^{[30]}$ observaram que a estabilidade térmica dos compósitos de PP com 3\% de argila organofilica e compatibilizante (1,5-12\% em peso) foi melhorada quando comparada ao PP puro. Yu-Hai et al. ${ }^{[31]}$ verificaram uma melhora significante na $\mathrm{T}_{\text {inicial }}$ de nanocompósitos de PP com 5 e $10 \%$ em massa de argila organofílica, respectivamente. Foi atribuída essa melhora ao efeito de barreira provocado pelas argilas

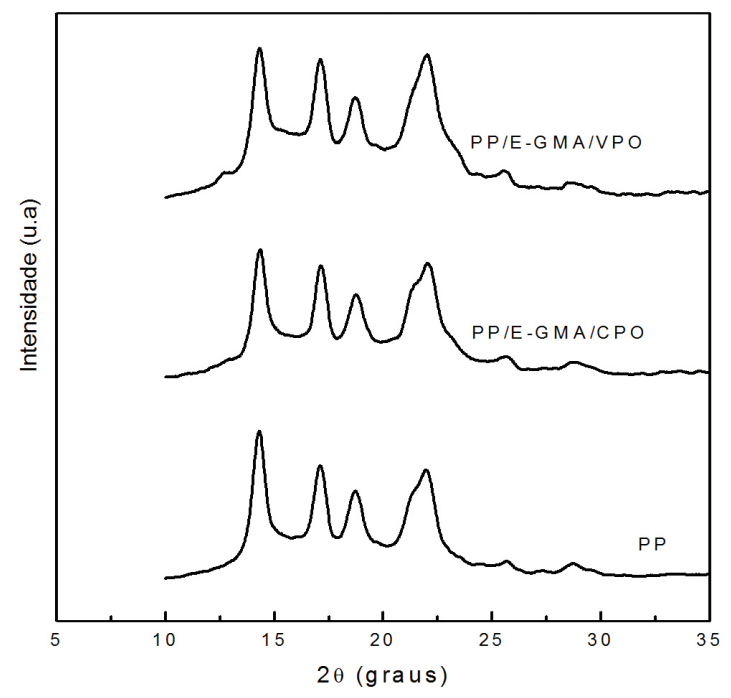

Figura 7. Difratogramas de raios-X do PP e dos sistemas PP/EGMA/argila.

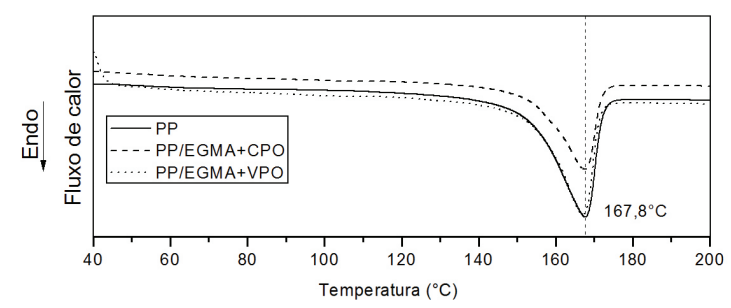

Figura 8. Curvas de DSC do PP puro e dos sistemas PP/E-GMA/ CPO e PP/E-GMA/VPO.

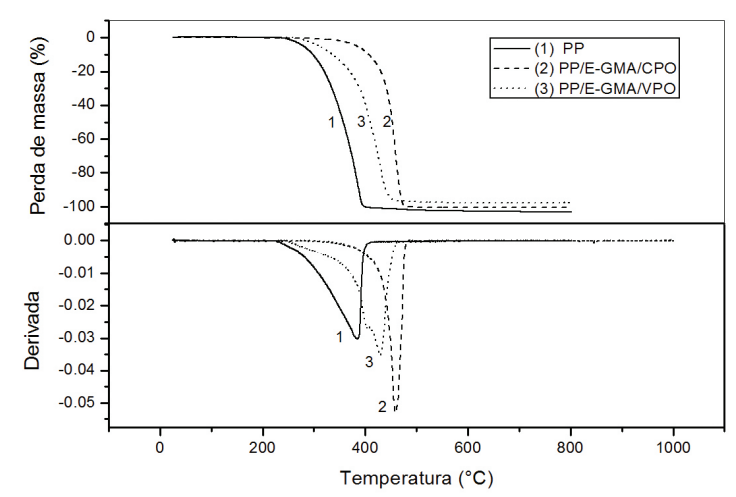

Figura 9. Curvas termogravimétricas do PP e dos sistemas PP/EGMA/CPO e PP/E-GMA/VPO.

organofílicas, dificultando a difusão de produtos voláteis e também do calor através do polímero.

\section{Propriedades mecânicas}

Ensaios de tração e impacto

A Tabela 7 apresenta os resultados do módulo de elasticidade, resistência à tração e resistência ao impacto do PP e dos sistemas PP/E-GMA/CPO e PP/E-GMA/ VPO.

Observa-se que os sistemas PP/E-GMA/argila apresentaram valores do módulo, considerando os erros 
Tabela 7. Propriedades mecânicas de PP e dos sistemas PP/E-GMA/argila.

\begin{tabular}{lccc}
\multicolumn{1}{c}{ Amostras } & Módulo de Elasticidade (MPa) & Resistência à Tração (MPa) & Resistência ao Impacto Izod (J/m) \\
\hline PP & $1361 \pm 46,0$ & $29,5 \pm 0,3$ & $28,63 \pm 3,0$ \\
PP/E-GMA/CPO & $1402 \pm 59,6$ & $27,3 \pm 0,4$ & $25,71 \pm 1,2$ \\
PP/E-GMA/VPO & $1351 \pm 65,9$ & $28,7 \pm 0,7$ & $26,07 \pm 1,5$ \\
\hline
\end{tabular}

experimentais, iguais ou ligeiramente superiores ao PP puro. Este comportamento significa que a rigidez do PP não foi alterada nem com a presença da argila organofilizada e nem com a presença do compatibilizante. Rodrigues et al. ${ }^{[18]}$ verificaram comportamento similar ao apresentado nesta pesquisa mesmo utilizando compatibilizantes e argila diferentes.

Os resultados de resistência à tração dos sistemas PP/E-GMA/CPO e PP/E-GMA/VPO apresentaram valores ligeiramente inferiores ao do PP puro. Este comportamento é importante uma vez que não foram deterioradas as propriedades de resistência à tração da matriz polimérica, conforme também observado por Rodrigues $^{[32]}$.

Os resultados de resistência ao impacto obtidos dos sistemas PP/E-GMA/CPO e PP/E-GMA/VPO, considerando os erros experimentais, foram semelhantes. Este resultado também foi observado por outros autores. Paiva et al. ${ }^{[33]}$ observaram que os valores encontrados de resistência ao impacto de nanocompósitos PP/ montmorilonita organofílica foram muito semelhantes e concluíram que a formação de nanocompósitos não proporcionou melhorias na propriedade de resistência ao impacto do polipropileno. Liu e $\mathrm{Wu}^{[34]}$ mostraram em seus estudos com nanocompósitos de PP/compatibilizante/ argila modificadas que a resistência ao impacto foi praticamente constante.

\section{Conclusões}

Neste trabalho, dois tipos de argila bentonita (verde e cinza) provenientes de Cubati-PB foram purificadas e organofilizadas em escala piloto e aplicadas no desenvolvimento de nanocompósitos poliméricos. As argilas foram caracterizadas por análise granulométrica por difração a laser (AG), análise química por Fluorescência de raios X (EDX), DRX e IV. Os resultados de AG mostraram que houve uma redução significativa no teor de areia, enquanto que a análise química por EDX mostrou que houve redução no teor de sílica livre na forma de quartzo, confirmando que o processo de purificação das argilas foi eficiente. As análises da argilas cinza e verde purificadas e organifilizadas (CPO e VPO) evidenciaram a presença do tensoativo na argila, indicando que as argilas CP e VP foram organofilizadas com sucesso. Os sistemas foram caracterizados por DRX, TG, DSC e propriedades mecânicas. O sistema PP/E-GMA/VPO apresentou a estrutura de um microcompósito, enquanto que o sistema PP/E-GMA/CPO formou um nanocompósito com estrutura intercalada em função da argila $\mathrm{CP}$ possuir maior teor de $\mathrm{SiO}_{2}$ que a VP e, consequentemente, maior grau de adsorção das cadeias de EO do tensoativo no $\mathrm{SiO}_{2}$, o que resultou em maior expansão desta argila, facilitando a intercalação das cadeias de PP entre as suas lamelas. Devido a uma maior dispersão da argila CPO, o nanocompósito PP/E-GMA/CPO foi o que apresentou maior estabilidade térmica e módulo de elasticidade. As demais propriedades mecânicas e a temperatura de fusão não foram significativamente alteradas quando comparadas com as do PP puro.

\section{Agradecimentos}

Os autores agradecem à Braskem pelo PP, à Arkema pelo compatibilizante E-GMA, à Oxiteno pelo tensoativo, ao Conselho Nacional de Desenvolvimento Científico e Tecnológico (CNPq) e à Coordenação de Aperfeiçoamento de Pessoal de Nível Superior (CAPES) pelo apoio financeiro.

\section{Referências Bibliográficas}

1. Deenadayalan, E.; Vidhate, S. \& Lele, A. - Polym. Int., 55, p.1270 (2006). http://dx.doi.org/10.1002/pi.2077

2. Kaempfer, D.; Thomann, R. \& Mulhaupt, R. - Polymer, 43, p.2909 (2002). http://dx.doi.org/10.1016/S00323861(02)00113-1

3. Vaia, R. A.; Ishii, H. \& Giannelis, E. P. - Chem. Mater., 5. p.1694 (1993). http://dx.doi.org/10.1021/cm00036a004

4. Vaia, R. A.; Jandt, K. D.; Kramer, E. J. \& Giannelis, E. P. - Macromolecules, 28, p.8080 (1995). http://dx.doi. org/10.1021/ma00128a016

5. Amorim, L. V. - "Melhoria, proteção e recuperação da reologia de fluidos hidroargilosos para uso na perfuração de poços de petróleo", Tese de Doutorado, Universidade Federal de Campina Grande, Brasil (2003).

6. Souza Santos, P. - "Tecnologia de argilas", Edgard Blücher, São Paulo (1992).

7. Iain, K. B.; Homer, J.; Monique, A. M. L. \& Willian, R. M. - Polyhedron, 14, p.2511 (1995).

8. Ferreira, H. S. - "Obtenção de argilas organofilicas purificadas para uso em fluidos de perfuração base óleo", Dissertação de Mestrado, Universidade Federal de Campina Grande, Brasil (2005).

9. Ferreira, H. S.; Menezes, R. R.; Ferreira, H. S.; Martins, A. B.; Neves, G. A. \& Ferreira, H. C. - Cerâmica, 54, p.77 (2008). http://dx.doi.org/10.1590/S036669132008000100011

10. Martins, A. B. - "Estudo de purificação de argilas esmectiticas por uso de hidrociclonagem", Dissertação de Mestrado, Universidade Federal de Campina Grande, Brasil (2009).

11. Shen, Y. H. - Chemosphere, 44, p.989 (2001). http://dx.doi. org/10.1016/S0045-6535(00)00564-6

12. Yuang, P. C. \& Shen, Y. H. - J. Colloid. Interf. Sci., 285, p.443 (2005). PMid:15837458. http://dx.doi.org/10.1016/j. jcis.2004.12.056 
13. García-López, D.; Picazo, O.; Merino, J. C. \& Pastor, J. M. - Eur. Polym. J., 39, p.945 (2003). http://dx.doi. org/10.1016/S0014-3057(02)00333-6

14. Xu, W.; Liang, G.; Zhai, H.; Tang, S.; Hang, G. \& Pan, E. - Eur. Polym. J., 39, p.1467 (2003). http://dx.doi. org/10.1016/S0014-3057(03)00015-6

15. Tidjani, A.; Wald, O.; Pohl, M.; Hentschel, M. P. \& Schartel, B. - Polym. Degrad. Stabil., 82, p.133 (2003). http://dx.doi. org/10.1016/S0141-3910(03)00174-5

16. Costa, J. M. R.; Silva, I. A.; Ferreira, H. S.; Menezes, R. R.; Neves, G. A.; Ferreira H, C. - Cerâmica, 58, p.419 (2012). http://dx.doi.org/10.1590/S0366-69132012000400002

17. Ferreira, H. S. - "Otimização do processo de organofilização de bentonitas visando seu uso em fluidos de perfuração não aquosos", Tese de Doutorado, Universidade Federal de Campina Grande, (2009).

18. Rodrigues, A. W. B.; Brasileiro, M. I.; Araújo, W. D.; Araújo, E. M.; Neves, G. A. \& Mélo, T. J. A. - Polímeros, 17, p.219 (2007).

19. Machado, J. C. - "Reologia de fluidos: aplicação na engenharia de petróleo", Petrobrás, Salvador (1983).

20. Menezes, R. R.; Melo, L. R. L.; Fonseca, F. A. S.; Ferreira, H. S.; Martins, A. B. \& Neves, G. A. - Rev. Eletrônica Mater. Proc., 3, p.36 (2008).

21. Campos, L. F. A. - "Composições de argilas bentoníticas para utilização em fluidos de perfuração de poços de petróleo", Tese de Doutorado, Universidade Federal de Campina Grande, Brasil (2007).

22. Ramos Filho, F. G.; Mélo, T. J. A.; Rabello, M. S. \& Silva, S. M. L. - Polym. Degrad. Stabil., 89 p.383 (2005). http:// dx.doi.org/10.1016/j.polymdegradstab.2004.12.011

23. Burmistr, M. V.; Sukhyy, K. M.; Shilov, V. V.; Pissis, P.; Spanoudak, A.;. Sukha, I. V.; Tomilo, V. I. \& Gomza, Y. P. - Polymer, 46, p.12226, (2005). http://dx.doi. org/10.1016/j.polymer.2005.10.094
24. Perrin-Sarazin, F.; Tom-That, M. T.; Bureau, M. N. \& Denault, J. - Polymer, 46, p.11624 (2005). http://dx.doi. org/10.1016/j.polymer.2005.09

25. Oliveira Junior, A. R. - "Obtenção de nanocompósitos polipropileno-argila compatibilizados com organossilanos", Tese de Doutorado, Universidade Estadual de Campinas, Brasil (2006).

26. Lai, S. M.; Chen, W. C. \& Zhu, X. S. - Compos.: Part. A, 40, p.754, (2009). http://dx.doi.org/10.1016/j. compositesa.2009.03.006

27. Raka, L.; Bogoeva-Gaceva, G.; Loos. J. - J. Therm. Anal. Calorim., 100, p.629 (2010). http://dx.doi.org/10.1007/ s10973-009-0545-y

28. Dal Castell, C. - "Estudo comparativo na obtenção de nanocompósitos de polipropileno/montmorilonita utilizando diferentes rotas de síntese”, Tese de Doutorado, Universidade Federal do Rio Grande do Sul, Brasil (2012).

29. Libano, E. V. D. G.; Visconte, L. L. Y. \& Pacheco, E. B. A. V. - Polímeros, 22, p.430 (2012).

30. Ding, C.; HE, H.; Jia, D. \& Guo, B. C. - Polym Compos., 29, p.698 (2008). http://dx.doi.org/10.1002/pc.20154

31. Yu-Hai, S.; Yuan-Fang, L.; De-Min, J. - Polym. Compos., 29, p.357 (2008). http://dx.doi.org/10.1002/ pc. 20395

32. Rodrigues, A. W. B. - “Organofilização de argilas bentonitas e aplicação no desenvolimento de nanocompósitos com matriz de polipropileno", Tese de Doutorado, Universidade Federal de Campina Grande, Brasil, (2009).

33. Paiva, L. B.; Morales, A. R. \& Guimarães, T. R. - Polímeros, 16, p.136 (2006).

34. Liu, X. \& Wu, Q. - Polymer, 42, p.10013 (2001). http:// dx.doi.org/10.1016/S0032-3861(01)00561-4

Enviado: Set. 3, 2013

Reenviado: Mar. 11, 2014

Aceito: Mar. 12, 2014 